\title{
Spiro-oxazine photochromic fiber optical switch
}

\author{
Yanyi Huang,, a) Liang, Joyce K. S. Poon, Yong Xu, Reginald K. Lee, and Amnon Yariv \\ Division of Engineering and Applied Science, California Institute of Technology, Pasadena, \\ California 91125
}

(Received 15 November 2005; accepted 27 February 2006; published online 2 May 2006)

A photochromic all-optical switch in telecommunication-grade fiber is fabricated by filling a fiber Bragg grating Fabry-Pérot resonator with a spiro-oxazine solution. The narrow linewidth of the resonator allows for a high sensitivity of the resonance wavelengths to the index change. The switch is controlled by low intensity UV light exposure, and operation at infrared telecommunication wavelengths is demonstrated. The switching speed on the order of minutes has been achieved.

(C) 2006 American Institute of Physics. [DOI: 10.1063/1.2200148]

All-optical photonic switches, which use light to control and gate the propagation of optical pulses, can have a significant impact on molecular photonics, optical computing, and networking. For current all-optical switching technologies, the major hurdles that limit their widespread application are low device responsivities and high optical intensities required to achieve the switching. In this letter, we report an all-optical switch that combines a "smart" fiber-based photonic structure with photochromic materials, ${ }^{1}$ and demonstrate nearly complete on-off switching under low intensity UV light exposure.

Photochromic compounds (including spiroheterocyclic compounds $^{2-5}$ such as spiropyrans ${ }^{3}$ and spiro-oxazines ${ }^{4}$ ) have a reversible response to light exposure and are ideal for optical switching applications. For example, spiro-oxazines have recently attracted much attention due to the high yield cleavage of spiro-C-O bond under ultraviolet (UV) exposure and the nearly total recovery of the back reaction after UV exposure. ${ }^{4,5}$ A photochromic optical switch can be based on either the conformational changes ${ }^{6,7}$ or the absorption changes ${ }^{8,9}$ of the chromophores. For spiro-oxazines, the colorless closed form [I, Fig. 1(a)] dominates the ground state equilibrium state while deep blue colored open form (II) is preferred under UV exposure, causing a drastic increase in absorption around $600 \mathrm{~nm}$. However, for most telecommunication applications, the working wavelengths (e.g., around $1500 \mathrm{~nm}$ ) are far away from the absorption band of the photochromic materials. Hence, a photochromic switch at these wavelengths must exploit changes in other optical properties, besides absorption, resulting from the photochromic reaction. For the fiber-based all-optical switch reported here, we adopt the most direct approach, ${ }^{10-12}$ which is to utilize the refractive index changes induced by the changes in the electronic delocalization in the chromophore. $^{13,14}$

5-chloro-1,3-dihydro-1,3,3-trimethylspiro-[2H-indole2, $3^{\prime}-(3 \mathrm{H})$ phenanthr[9,10-b](1,4)oxazine $]$ (SPO), whose structure is shown in Fig. 1, is chosen as the working material for the switch. Under UV exposure, the ring open reaction produces the merocyanine form II. The charge transfer transition of this merocyanine chromophore changes the material absorptivity around $600 \mathrm{~nm}$ and consequently modifies the refractive index in the infrared (IR) wavelengths. After

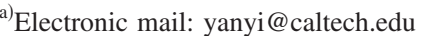

the UV exposure, the merocyanine structure returns to the spiroconformation (I) and the material refractive index returns to the original value.

To utilize this photoinduced index change to control light propagation, we put this reaction in a Fabry-Pérot (FP) microresonator. An optical resonator is highly sensitive to

(a)

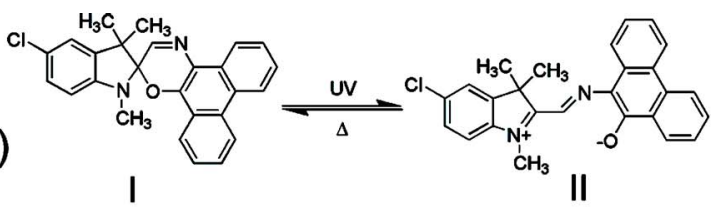

(b)
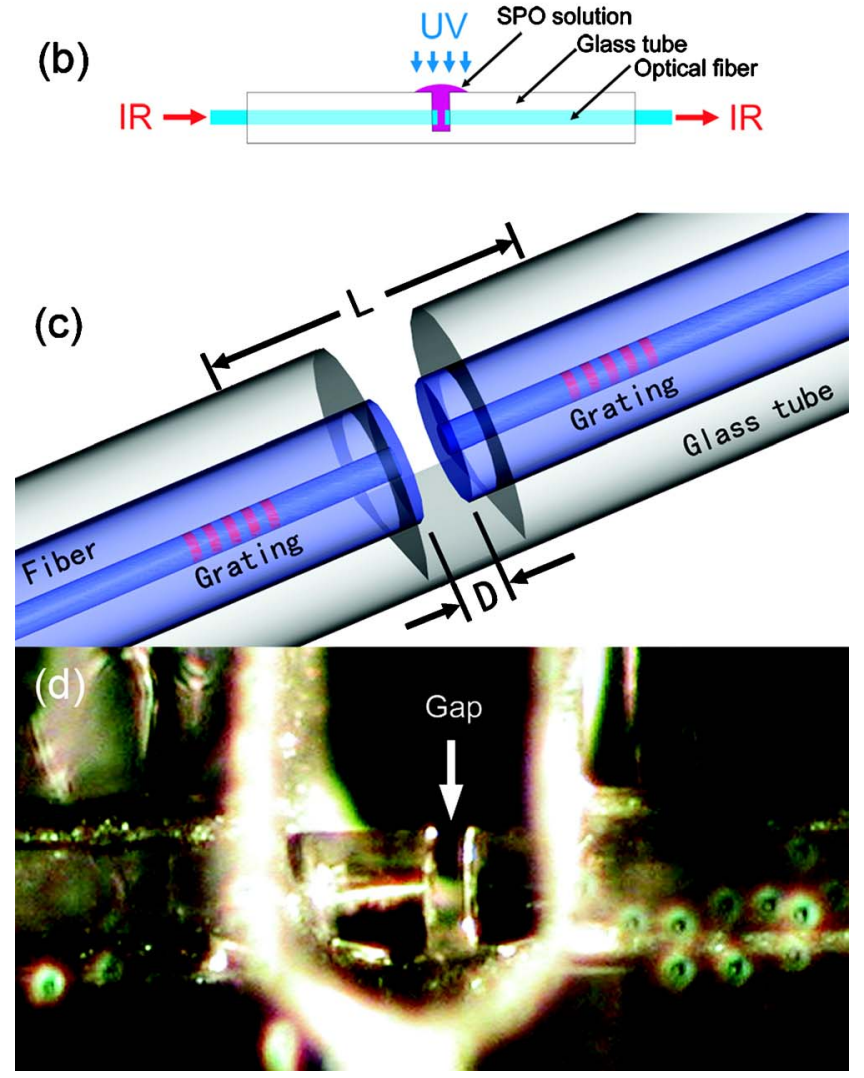

FIG. 1. (Color online) (a) Molecular structures of the closed state (I) and the open state (II) of the SPO chromophore. (b) The schematic of the fiber optical switch device. (c) The two fibers are separated by a gap of length $D$. The gratings are separated by a length $L$. (d) An optical microscope photograph of the photochromic fiber switch device. The diameter of the fiber is $125 \mu \mathrm{m}$ and the gap distance $D$ is $40 \mu \mathrm{m}$. 


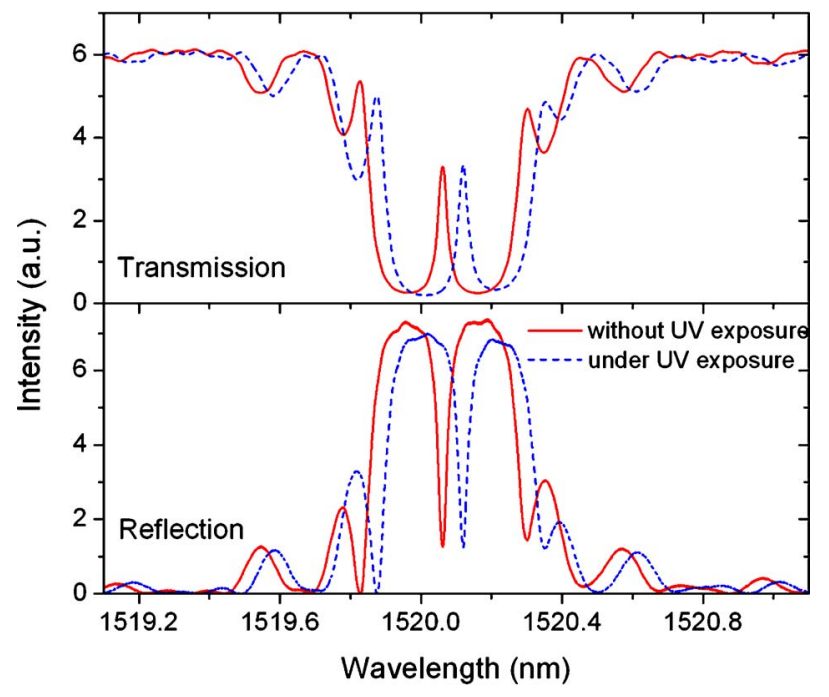

FIG. 2. (Color online) Transmission (top) and reflection (bottom) spectra of the fiber optical switch with and without the UV light exposure.

changes in the refractive index of the cavity because an index change alters the intracavity optical path length, thus modifying the resonance condition of the cavity. Using this principle, FP resonators have recently been used in fiber for sensing applications. ${ }^{15-17}$

A drawback to using SPO is that the photochromic reaction is slow in polymer blends and is prohibited in crystal form $^{18}$ as the conformation change is impeded by the solid phase. Therefore, we combine the SPO liquid solution with optical fibers. Two $2.5 \mathrm{~mm}$ long Bragg gratings with reflection bands around $1520 \mathrm{~nm}$ and reflectivity $\sim 95 \%$ are written in the core of an SMF-28 single-mode fiber by a $\mathrm{KrF}$ laser $(248 \mathrm{~nm})$ using quartz phase mask. The two Bragg gratings serve as the end mirrors of the FP cavity. The fiber was cleaved in the middle of the two grating reflectors, and the two halves are fixed in a glass tube with a gap of length $D$ in between them [Fig. 1(c)]. The separation of the two gratings is $L$. In our device, $D=40 \mu \mathrm{m}$ and $L=1.4 \mathrm{~mm}$. An opening is cut at the top of the glass tube so that the SPO solution can be deposited into the air gap [Figs. 1(b)-1(d)]. To avoid reflection at the interface between the fiber ends and the solution, we use fused silica index matching fluid as the solvent for the SPO powder. The concentration of the solution is $4 \times 10^{-3} \mathrm{~mol} \mathrm{dm}^{-3}$.

The fiber and the solution in the gap contribute to the total optical length of the FP cavity. Under UV exposure, the refractive index of the SPO solution increases and therefore extends the optical length of the FP cavity. The transmission and reflection spectra of the device are shown in Fig. 2. The transmission spectrum shows a stop band centered at $1520.05 \mathrm{~nm}$ with bandwidth $\sim 0.5 \mathrm{~nm}$. The reflection spectrum is complementary to the transmission spectrum. From Fig. 2, the loaded quality $(Q)$ factor of the device, which is indicative of the total resonator loss, is $4.5 \times 10^{4}$, and the free spectral range (FSR) of the FP resonator is $0.23 \mathrm{~nm}$.

The resonance wavelengths are redshifted when the photochromic solution is exposed by $365 \mathrm{~nm}$ UV light with an intensity of $10 \mathrm{~mW} / \mathrm{cm}^{2}$ in ambient conditions. Theoretical fitting of the spectrum under UV exposure shows that the $0.06 \mathrm{~nm}$ redshift of the resonance wavelength corresponds to a refractive index increase of $4.0 \times 10^{-3}$ in the photochromic solution. Thus, the responsivity of the optical switch $\Delta \lambda / \Delta n$ Downloaded 26 May 2006 to 131.215.225.175. Redistribution subje

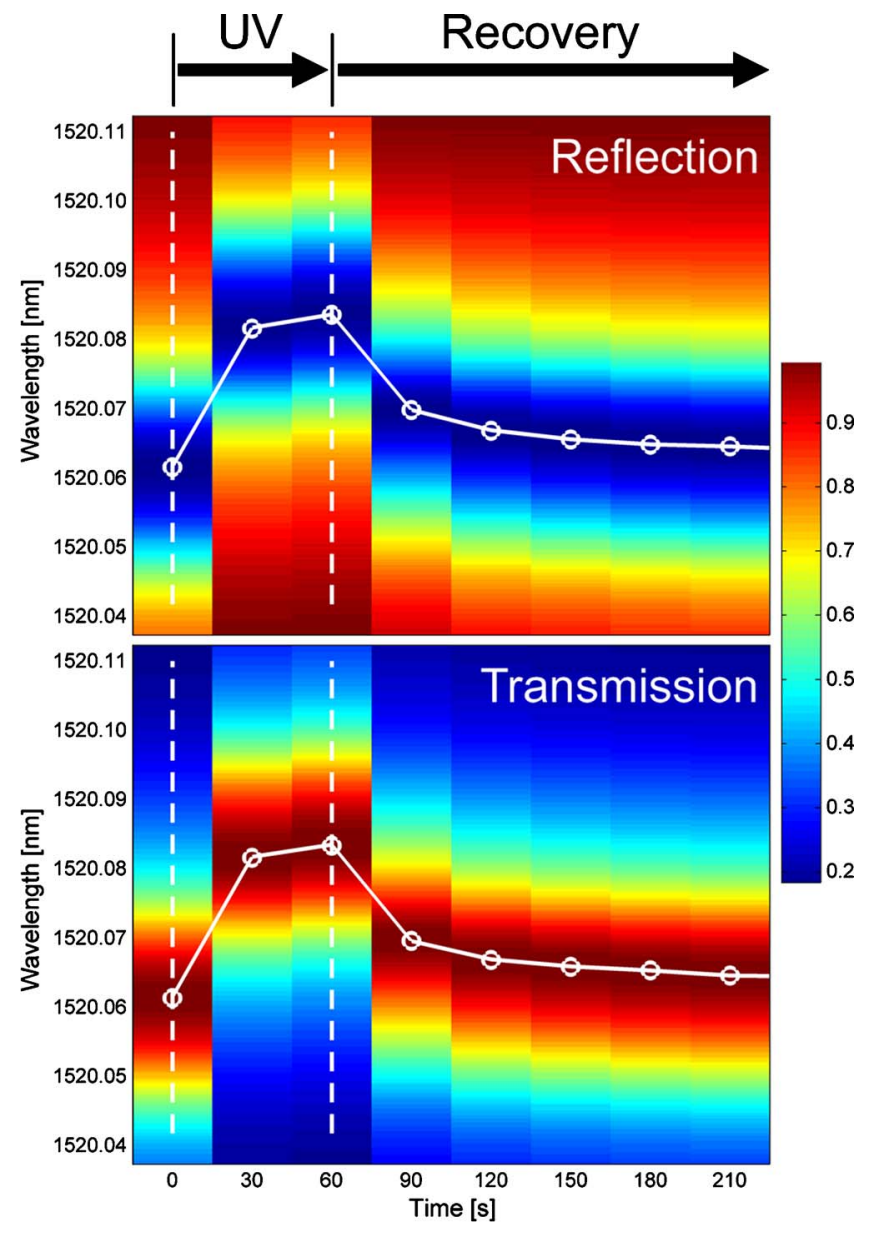

FIG. 3. (Color online) The time-dependent shift of the resonance wavelength during and after the UV exposure. The spectra around the resonance peak/notch are plotted as color map diagrams. The warmer color indicates a higher intensity while the colder a lower intensity, as shown in the color bar. The center of the resonance peaks/notches is plotted as circles. The UV exposure period and the recovery are also labeled.

is $15 \mathrm{~nm}$. For this high- $Q$ resonator device, the resonance peaks are narrow (linewidth of $0.034 \mathrm{~nm}$ ) such that a $0.06 \mathrm{~nm}$ shift is sufficient to move the resonance wavelengths over the width of the peaks. Therefore, the resonance wavelengths without UV exposure can become completely off-resonance during the UV exposure.

Figure 3 is the time-dependent transmission and reflection spectra of the photochromic switch during and after the UV exposure. The time step between the spectra is $30 \mathrm{~s}$. The UV exposure takes place within the first $60 \mathrm{~s}$. Only the spectra around one resonance peak/notch are shown in the figure. In the first $60 \mathrm{~s}$, the resonance peak/notch redshifts, after which it shifts back. Within $150 \mathrm{~s}$ after the absence of UV irradiation, the spectrum shift recovers $\sim 80 \%$. Eventually the resonance peak/notch returns to the original wavelength.

The bistability in SPO can be used to construct an optical switch. Light at the resonance wavelength before an UV exposure is transmitted through the device and is thus at the "on" state in the transmission port and the "off" state in the reflection port. Under UV exposure, the resonance wavelength shifts, and the two states switch, so the off state is in the transmission while the on state is in the reflection. The characteristics of this photochromic optical switch are shown in Fig. 4. The laser light is fixed at $1520.06 \mathrm{~nm}$, coinciding with the resonance wavelength without the UV exposure in to AlP license or copyright, see http://apl.aip.org/apl/copyright.jsp 


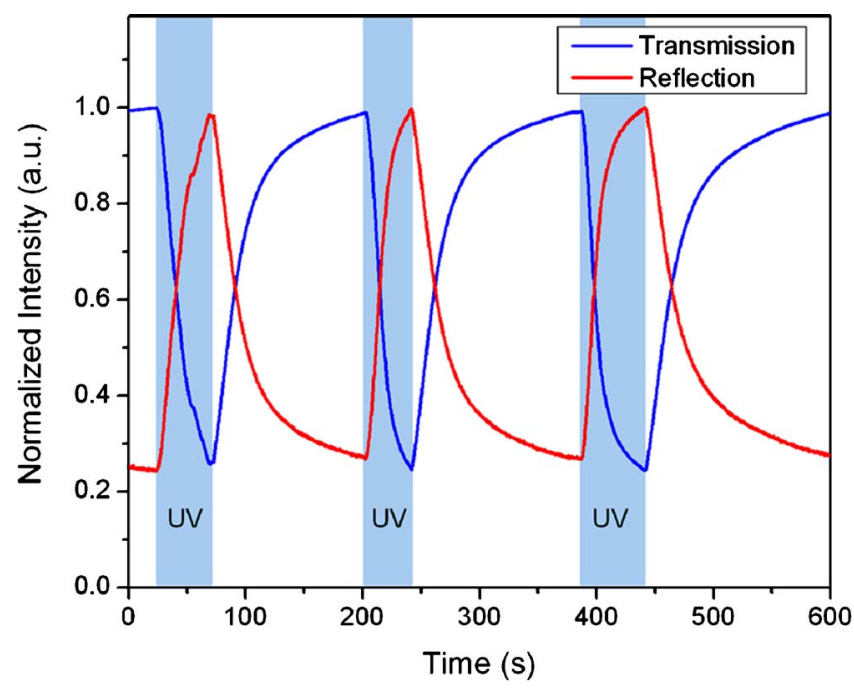

FIG. 4. (Color online) Modulation of the IR signal light at $1520.06 \mathrm{~nm}$ due to the photochromism induced by the UV exposure.

Fig. 2. By controlling the UV light exposure, this hybrid fiber device demonstrates reversible switching in both the transmission and reflection ports of the device. Due to the incomplete photochromic reaction and the possible photobleaching and photo-oxidation of the chromophores, the UV exposure time required for switching increases and the switch may degrade with the number of switching cycles. However, under ambient conditions, our prototype device has successfully completed dozens of switching cycles.

Compared with other all-optical switching mechanisms, which usually require high power $\left(\sim 10^{6} \mathrm{~W} / \mathrm{cm}^{2}\right)$ light pulses $^{19}$ to control the switch, our device requires a significantly lower intensity for the control light $\left(\sim 10 \mathrm{~mW} / \mathrm{cm}^{2}\right)$. The switching times are about $10 \mathrm{~s}$ during the UV exposure and $100 \mathrm{~s}$ without the UV exposure in ambient conditions. The switching speeds are limited by the photochromic reaction rate of SPO in nonpolar solvents. However, this response time is commensurate with related reports and can be optimized by the molecular engineering of the photochromic materials. $^{20}$

The authors thank the National Science Foundation, the Defense Advanced Research Projects Agency (Dr. R. Athale and Dr. D. Honey), and the Hughes Research Laboratories LLC (Dr. W. Ng) for their financial support. One of the authors (J.K.S.P.) is grateful for the support of the Natural Sciences and Engineering Research Council of Canada.

${ }^{1}$ Photochromism, Molecules and Systems, revised ed., edited by H. Durr and H. Bouas-Laurent (Elsevier, Amsterdam,, 2003).

${ }^{2}$ V. I. Minkin, Chem. Rev. (Washington, D.C.) 104, 2751 (2004).

${ }^{3}$ M. Rini, A.-K. Holm, E. T. J. Nibbering, and H. Fidder, J. Am. Chem. Soc. 125, 3028 (2003).

${ }^{4}$ A. K. Chibisov and H. Gorner, Phys. Chem. Chem. Phys. 3, 424 (2001).

${ }^{5}$ G. Berkovic, V. Krongauz, and V. Weiss, Chem. Rev. (Washington, D.C.) 100, 1741 (2000).

${ }^{6}$ S. Z. Janicki and G. B. Schuster, J. Am. Chem. Soc. 117, 8524 (1995).

${ }^{7}$ N. Tamaoki, E. V. Keuren, H. Matsuda, K. Hasegawa, and T. Yamaoka, Appl. Phys. Lett. 69, 1188 (1996).

${ }^{8}$ X. Guo, D. Zhang, G. Zhang, and D. Zhu, J. Phys. Chem. B 108, 11942 (2004).

${ }^{9}$ M. Saito, A. Honda, and K. Uchida, J. Lightwave Technol. 21, 2255 (2003).

${ }^{10}$ J. Hobley, H. Fukumura, and M. Goto, Appl. Phys. A: Mater. Sci. Process. 69, S945 (1999).

${ }^{11}$ J.-W. Kang, J.-J. Kim, and E. Kim, Appl. Phys. Lett. 80, 1710 (2002).

${ }^{12}$ Z.-Z. Gu, T. Iyoda, A. Fujishima, and O. Sato, Adv. Mater. (Weinheim, Ger.) 13, 1295 (2001).

${ }^{13}$ J. Biteau, F. Chaput, K. Lahlil, J.-P. Boilot, G. M. Tsivgoulis, J.-M. Lehn, B. Darracq, C. Marois, and Y. Levy, Chem. Mater. 10, 1945 (1998).

${ }^{14}$ C. Bertarelli, A. Bianco, F. D'Amore, M. C. Gallazzi, and G. Zerbi, Adv. Funct. Mater. 14, 357 (2004).

${ }^{15}$ M. Han, Y. Zhang, F. Shen, G. R. Pickrell, and A. Wang, Opt. Lett. 29, 1736 (2004).

${ }^{16}$ B. Yu, G. Pickrell, and A. Wang, IEEE Photonics Technol. Lett. 16, 2296 (2004).

${ }^{17}$ Y. Zhu and A. Wang, IEEE Photonics Technol. Lett. 17, 447 (2005).

${ }^{18}$ M. Suzuki, T. Asahi, and H. Masuhara, Phys. Chem. Chem. Phys. 4, 185 (2002).

${ }^{19}$ For example, see H. Kanbara, M. Asobe, K. Kubodera, T. Kaino, and T. Kurihara, Appl. Phys. Lett. 61, 2290 (1992).

${ }^{20}$ R. A. Evans, T. L. Hanley, M. A. Skidmore, T. P. Davis, G. K. Such, L. H. Yee, G. E. Ball, and D. A. Lewis, Nat. Mater. 4, 249 (2005). 